\title{
Neue „Choosing wisely“ Empfehlungen zu unangemessenen medizinischen Interventionen: Sicht von Schweizer Hausärzten
}

\author{
Neuner-Jehle, Stefan ; Senn, Oliver ; Rosemann, Thomas
}

\begin{abstract}
Aim: As part of the "Choosing wisely" campaign expert-driven recommendations of inappropriate interventions which lead to overdiagnosis and overtreatment are being published. The aim of our work was to describe an innovative method for developing recommendations together with general practitioners (GPs) and to compare the results with the "Choosing wisely" campaign lists as well as with the Swiss "Smarter medicine" shortlist. Methods: We asked 109 GPs who attended a medical education conference to form groups (of 5 to 7 GPs each) and develop three interventions that are relevant to their work and should be avoided. We then compared the most frequently suggested interventions with those of the "Choosing wisely" campaign list and the "Smarter medicine" campaign shortlist. Finally, we asked the Swiss Young GPs Association (JHaS) members for additional suggestions. Results: Five groups suggested avoidance of check-up examinations, especially in younger or asymptomatic individuals. Further unnecessary interventions, which were mentioned with similar frequency, included resting or exercise electrocardiography in asymptomatic individuals and cholesterol analysis in individuals older than 75 years, or statin therapy in primary prevention and/or high age. Four groups suggested avoiding arthroscopy or magnetic resonance imaging of the knee joint after an injury (in the absence of joint instability or blockade), and three groups recommended to avoid imaging diagnostic procedures in patients with unspecific headache (in the absence of red flags). There was no consistency between interventions of the GPs' list and the list of the Swiss "Smarter medicine" official campaign. The interventions that were most frequently mentioned by the GPs are also present on the lists issued by are present on lists of medical societies that have joined the "Choosing wisely" campaign. The response rate from the Swiss Young GPs association members was impressively low. Conclusion: The perspective of users (GPs) is crucial for the development of lists of potentially inappropriate interventions. In order to enhance the degree of identification with and adherence to the recommendations. The interventions suggested in our study could lead to further recommendations on interventions to be avoided in primary care, ideally in collaboration with the "Smarter medicine" campaign. Empathic communication with patients about harms and benefits of potentially inappropriate interventions is crucial for the implementation of this policy.
\end{abstract}

DOI: https://doi.org/10.1016/j.zefq.2016.09.001

Posted at the Zurich Open Repository and Archive, University of Zurich ZORA URL: https://doi.org/10.5167/uzh-130351

Journal Article

Accepted Version

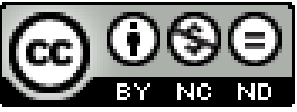

The following work is licensed under a Creative Commons: Attribution-NonCommercial-NoDerivatives 4.0 International (CC BY-NC-ND 4.0) License. 
Originally published at:

Neuner-Jehle, Stefan; Senn, Oliver; Rosemann, Thomas (2016). Neue „Choosing wisely“ Empfehlungen zu unangemessenen medizinischen Interventionen: Sicht von Schweizer Hausärzten. Zeitschrift für Evidenz, Fortbildung und Qualität im Gesundheitswesen, 118-119:82-86.

DOI: https://doi.org/10.1016/j.zefq.2016.09.001 


\section{Neue "Choosing wisely" Empfehlungen zu unangemessenen medizinischen Interventionen: Sicht von Schweizer Hausärzten}

New "choosing wisely" recommendations of inappropriate interventions: the perspective of Swiss general practitionners

\section{Autoren/Affiliationen:}

Stefan Neuner-Jehle1, stefan.neuner-jehle@usz.ch (corresponding author) OliverSenn ${ }^{1}$,oliver.senn@usz.ch

Thomas Rosemann ${ }^{1}$, thomas.rosemann@usz.ch

(1) Institut für Hausarztmedizin Zürich, Pestalozzistrasse 24, CH-8091 Zürich

\section{Keywords in English:}

Choosing wisely; inappropriate interventions; overdiagnosis; overtreatment; health services research

\section{Keywords in German:}

Gemeinsam klug entscheiden; unangemessene Interventionen; Überdiagnose; Überbehandlung; Versorgungsforschung

\section{Abstract in English:}

Aim: As part of the "Choosing wisely" campaign expert-driven recommendations of inappropriate interventions which lead to overdiagnosis and overtreatment are being published. The aim of our work was to describe an innovative method for developing recommendations together with general practitioners (GPS) and to compare the results with the "Choosing wisely" campaign lists as well as with the Swiss "Smarter medicine" Shortlist. 
Methods: We asked 109 GPs who attended a Medical Education Conference to develop in groups (of 5 to 7 GPs each) three interventions to avoid which are relevant for their work. We then compared the most frequently suggested interventions with those of the "Choosing wisely" campaign list and the "Smarter medicine" campaign shortlist. Finally we asked the Swiss Young GPs Association (JHaS) members for additional suggestions. Results: Five groups suggested to avoid check-up examinations, especially in younger or asymptomatic individuals. Resting or exercise electrocardiography in asymptomatic individuals and cholesterol analysis in individuals older than 75 years, or statin therapy in primary prevention and/or high age were similarly frequently suggested recommendations to avoid. Four groups suggested to avoid arthroscopy or magnetic resonance imaging of the knee joint after an injury (in absence of joint instability or blockade), and three groups recommended to avoid imaging diagnostic procedures in patients with unspecific headache (in the absence of red flags). There was no accordance between interventions of the GPs' list and the list of the Swiss "Smarter medicine" official campaign. The most frequently suggested GPs' interventions are present on lists of medical societies joining the "Choosing wisely" campaign. The response rate from the Swiss Young GPs association members was impressively low.

Conclusion: For the development of lists of potentially inappropriate interventions the perspective of users (GPs) is crucial, in regard to the degree of identification with and adherence to the recommendations. The interventions suggested in our study could lead to further recommendations on what to avoid in primary care, optimally in collaboration with the "Smarter medicine" campaign. For the implementation an empathic communication with patients about harms and benefits of potentially inappropriate interventions is of utmost importance.

\section{Abstract in German:}

Ziel: Im Rahmen der "Choosing wisely"-Kampgagne werden Empfehlungen von Experten zu diagnostischen oder therapeutischen Interventionen publiziert, die unangemessen 
sind und zu Überdiagnose oder Überbehandlung führen. Ziel unserer Arbeit war, eine innovative Methode darzustellen, wie Empfehlungen mit praktizierenden Hausärzten entwickelt werden können, und die Resultate mit den Listen der "Choosing wisely"-Kampgagne und mit der Schweizerischen "Smarter medicine"-Kurzliste zu vergleichen.

Methoden: 109 niedergelassene Hausärzte, die an einer Kongress-Fortbildung teilnahmen, wurden gebeten, in Gruppen (zu 5-7 Ärzten pro Gruppe) drei für ihre Arbeit relevante Intervention zu entwickeln, die zu vermeiden seien. Die am häufigsten genannten Interventionen verglichen wir mit denjenigen der publizierten Listen der "Choosing wisely"-Kampagne und der Kurzliste der "Smarter Medicine"-Kampagne. Die Themenliste wurde ergänzt mit Vorschlägen, die von Mitgliedern des Verbandes Junger Hausärzte Schweiz (JHaS) erfragt wurden.

Resultate: Fünf Gruppen schlugen Check-up-Untersuchungen als verzichtbare Intervention vor, vor allem bei jüngeren oder asymptomatischen Individuen. Die Durchführung von Ruhe- oder Belastungs-Elektrokardiogrammen bei asymptomatischen Individuen und die Cholesterinmessung bei Individuen älter als 75 Jahre, oder die Statintherapie in der Primärprävention und/oder hohem Alter, waren ebenso häufig genannte verzichtbare Interventionen. Vier bzw. drei Gruppen nannten die Arthroskopie oder ein MRI des Kniegelenks nach Distorsion (in Abwesenheit von Gelenksinstabilität oder -blockade) bzw. die bildgebende Diagnostik bei Kopfweh (ohne Alarmzeichen) als verzichtbare Interventionen. Zwischen den Interventionen, die von Hausärzten vorgeschlagen wurde, und denjenigen auf der Liste der "Smarter medicine"-Kampagne bestand keinerlei Übereinstimmung. Hingegen fanden wir bei den am häufigsten vorgeschlagenen Interventionen Entsprechungen auf den Listen der "Choosing wisely" Partnergesellschaften. Die Rücklaufquote der JHaS-Mitglieder war eindrücklich niedrig.

Konklusion: Für die Entwicklung von Listen mit potentiell unangemessenen Interventionen ist die Perspektive der Anwender (praktizierende Ärztinnen und Ärzte) wichtig. Dadurch kann ein höherer Identifikationsgrad und Adhärenz der Anwender mit den Empfehlungen erwartet werden. Die in unserer Studie vorgeschlagenen 
Interventionen könnten, optimalerweise in Zusammenarbeit mit der "Smarter medicine"-Kampagne, zu weiteren Empfehlungen führen, was in der Grundversorgung besser zu unterlassen sei. Entscheidend für die Umsetzung ist eine einfühlsame Kommunikation mit dem Patienten zu Nutzen und Schaden von potentiell unangemessenen Interventionen.

\section{Danksagungen:}

Wir danken den teilnehmenden Ärztinnen und Ärzten für ihre konstruktiven Beiträge.

Funding:

This research did not receive any specific grant from funding agencies in the public, commercial, or not-for-profit sectors.

\section{Manuskript}

\section{Einführung}

\subsection{Der historische Hintergrund}

Studien zur Überversorgung als Ausdruck problematischer Versorgungsqualität werden seit den 1920er Jahren publiziert [1-5], und Studien zu medizinisch nicht gerechtfertigten regionalen Versorgungsunterschieden folgten um 1970 [6]. 1998 wurde eine "dringende Notwendigkeit, die Qualität der Gesundheitsversorgung zu verbessern", festgestellt [7][8]. Unter dem Schlagwort "Too much medicine" wurde schliesslich 2002 im British Medical Journal der öffentliche Diskurs darüber aufgenommen, dass in der medizinischen Versorgung Interventionen stattfinden, bei denen das Verhältnis von Nutzen zu Risiken ungünstig ist [9]. Bald folgten weitere Publikationen zur Überbehandlung, und nebst dem individuellen Schadenspotential wurden die ökonomischen Folgen einer unangemessenen Versorgung fokussiert: "Why too much medicine is making us sicker 
and poorer" [10]. Patienten und potentielle Teilnehmer ${ }^{1}$ von Screeningprogrammen wurden erstmals ermutigt, möglicherweise unangemessene Interventionen zu verweigern: "It is not wrong to say no" [11]. Konzepte wie Medikalisierung [12], Überdiagnose und Überbehandlung [13] führten zur Erkenntnis, dass „Weniger mehr sein kann“ („Less is more“) - ein Prinzip, das sich über die letzten Jahre, unter Einbezug der mächtigen Consumer's Health Organisation, von USA aus international unter dem Namen "Choosing wisely" [14] etabliert hat. Dabei ist nicht nur das "weise Auswählen" auf Patientenseite angesprochen, sondern auch die Qualität ärztlichen professionellen Verhaltens [15]. Ein Ziel ist zum Beispiel, die Kluft zwischen ethischem Anspruch von Ärzten und der unzureichenden Umsetzung der Prinzipien im Berufsalltag zu überwinden [16][17]. Die Empfehlungen dienen in erster Linie der Stimulation von Gesprächen zwischen Ärzten und Patienten über eine vernünftige, insbesondere ein Zuviel vermeidende Medizin [18]. Zusammenfassend ist es das Ziel all dieser Anstrengungen, unnötige oder gar schädliche medizinische Interventionen zu vermeiden und damit Effizienz und Qualität der medizinischen Versorgung zu verbessern.

Auch in der Schweiz gibt es Bereiche, in denen eine qualitativ inadäquate Versorgung vermutet wird. Die Schweizerische Gesellschaft für Allgemeine Innere Medizin (SGAIM) hat daher unter dem Namen "Smarter medicine" [19] eine Bewegung gestartet, die von inadäquaten Interventionen abrät: In einem ersten Schritt wurden aus der Fachliteratur 1'090 unangemessene medizinische Interventionen identifiziert. 158 davon betrafen den ambulanten Bereich der Allgemeinmedizin. Nach Ausschluss von unklar formulierten oder doppelten Nennungen wurden 38 Interventionen gelistet, die für eine Kurzliste von Empfehlungen in Frage kamen. 35 Experten (aus den Vorständen der Schweizerischen Gesellschaften für Allgemeine und für Innere Medizin sowie aus den entsprechenden universitären Instituten) bewerteten diese Vorschläge in einem mehrstufigen Delphi-Verfahren hinsichtlich Relevanz. Schliesslich gewichteten sie die verbleibenden 18 Interventionen nach Häufigkeit, Schadenspotential und Kosten. Aus den zehn Interventionen dieser Liste, die nach der Meinung der Experten am häufigsten in der

\footnotetext{
${ }^{1}$ Mit der männlichen Form ist jeweils gleichbedeutend auch die weibliche Form gemeint.
} 
Allgemeinmedizin vorkommen, wählte das Studienteam die fünf mit der höchsten Relevanz aus. Diese wurden schliesslich 2014 von der SGAIM als Shortlist publiziert [19][20](Tabelle 1). Weitere Empfehlungen, welche nun die stationäre Allgemeine Innere Medizin betreffen, sind von der SGAIM im Mai 2016 publiziert worden [19].

\subsection{Ziele der Arbeit}

Ziel unserer Arbeit war die Darstellung eines innovativen Entwicklungsprozesses zu Empfehlungen, auf welche unangemessenen Interventionen in der ambulanten Allgemeinmedizin besser zu verzichten sei. Für bisher publizierte Schweizer Empfehlungen in der Allgemeinmedizin zeichnen universitäre und/oder standespolitische "Opinion leaders" verantwortlich [19][20], während unser Fokus auf der direkten Befragung niedergelassener Allgemeinärzte lag, als Experten für die Praxisrelevanz von Empfehlungen. Ferner wollten wir prüfen, in welchem Mass sich die solchermassen generierten Empfehlungen von denjenigen der "Smarter medicine"-Liste unterschieden. Ausserdem stellten wir uns die Frage, ob sich diese Vorschläge auf den "Choosing wisely"-Listen verschiedener Fachgesellschaften wiederfinden. Des weiteren interessierte uns, wie die Verteilung zwischen diagnostischen und therapeutischen Interventionen unter den vorgeschlagenen Interventionen war. Schliesslich erhoben wir noch Empfehlungen von der jungen Schweizer Hausärztegeneration zum Thema.

\section{Material und Methoden}

Unter den Teilnehmern (TN) des 54. Ärztekongresses der "Lunge Zürich" in Davos (Januar 2015) nahmen 109 niedergelassene Allgemeinpraktiker (mittleres Alter 53 Jahre, SD 11.2; mittlere Arbeitsdauer in der Praxis 19 Jahre, SD 9.9) an drei seriell durchgeführten Workshops mit dem Titel "Choosing wisely"teil. Sämtliche 109 TN dieser drei Workshops waren einverstanden, an der Studie teilzunehmen. Im moderierten Workshop wurde das Prinzip der "Choosing wisely"- und "Smarter medicine"-Bewegungen dargestellt, die publizierten Empfehlungen wurden jedoch erst nach der Erhebung der Vorschläge unter 
den TN vorgestellt, um deren Vorschläge nicht zu beeinflussen. Wir baten die TN während des Workshops, drei Empfehlungen zu entwickeln, welche Interventionen zu vermeiden seien. Die Vorschläge sollten auf ihrer eigenen Praxiserfahrung basieren, und sich entweder auf diagnostische oder therapeutische Interventionen beziehen. Deren Häufigkeit und das Ungleichgewicht zwischen potentiellem Schaden und potentiellem Nutzen für den Patienten sollten relevant für ihre tägliche Arbeit sein. In Arbeitsgruppen von je 5-7 Ärzten (total 18 Gruppen) wurden die Empfehlungen erarbeitet und sich gegenseitig im Plenum präsentiert. Alle von den TN beschriebenen Themen wurden nach Häufigkeit gelistet, mit „Choosing wisely" Listen verschiedener Fachgesellschaften wie auch mit der Schweizer "Smarter medicine" Shortlist verglichen und nach diagnostischen versus therapeutischen Interventionen klassiert.

Am Kongress des Verbandes der Jungen Hausärzte Schweiz (JHaS) in Thun (April 2016) wurde der Kongressmappe ein Aufruf beigelegt, Empfehlungen abzugeben, welche Interventionen zu vermeiden seien. Derselbe Aufruf wurde im Mai 2016 in einem Newsletter allen Verbandsmitgliedern per E-mail verschickt (gesamthafte Anzahl Kongressbesucher plus Mitglieder rund 500).

\section{Resultate}

Die 18 TN-Gruppen entwickelten gesamthaft 44 Vorschläge (8 Gruppen mit je 3 Empfehlungen, 10 Gruppen mit je 2 Empfehlungen). Fünf Gruppen schlugen Check-up-Untersuchungen als verzichtbare Intervention vor, vor allem bei jüngeren oder asymptomatischen Individuen. Die Durchführung von Ruhe- oder Belastungs-Elektrokardiogrammen (EKG's) bei asymptomatischen Individuen und die Cholesterinmessung bei Individuen älter als 75 Jahre, oder die Statintherapie in der Primärprävention und/oder hohem Alter, waren ebenso häufig genannte Interventionen. Mit vier bzw. drei Gruppen-Nennungen wurden die Arthroskopie oder ein MRI des Kniegelenks nach Distorsion (in Abwesenheit von Gelenksinstabilität oder -blockade) und die bildgebende Diagnostik bei Kopfweh (ohne Alarmzeichen) genannt (Übersicht in 
Tabelle 2). Die weniger häufig (je 2 Nennungen) vorgeschlagenen Themen sind in Tabelle 3 dargestellt.

Zwischen den Themen, die von den TN am häufigsten vorgeschlagen wurden, und den Themen der "Smarter medicine" Shortlist gab es keine Übereinstimmung. Die fünf am häufigsten vorgeschlagenen Themen fanden sich auf "Choosing wisely" Listen verschiedener Fachgesellschaften wieder, die in den letzten 3 Jahren publiziert wurden (Tabelle 2). Vier von fünf der am häufigsten vorgeschlagenen Themen waren diagnostische Interventionen, während auf der "Smarter medicine" Shortlist diagnostische und therapeutische Interventionen gleich häufig vertreten sind. Unter den von unseren TN weniger häufig vorgeschlagenen Themen waren diagnostische und therapeutische Interventionen wiederum gleich häufig.

Aus der JHaS-Befragung kamen 5 Antworten (Rücklaufquote 1\%), die sowohl diagnostische (Check-up Untersuchungen, Routine-Urinkontrollen, Screening nach Vitamin D-Mangel und Prostata-Karzinom) als auch therapeutische (Statine, Protonenpumpenblocker) Themen umfassten.

\section{Diskussion}

Die von unserer Studienpopulation von niedergelassenen Allgemeinärzten vorgeschlagenen unangemessenen Interventionen unterschieden sich inhaltlich klar von der Themenwahl der "Smarter medicine"-Kampagne, deren Shortlist von einem akademisch und/oder standespolitisch engagierten Expertengremium definiert worden war. Bei der Entwicklung der Kampagne war auf der Basis der wissenschaftlichen Literatur zu potentiell unangemessenen Interventionen eine Auswahl getroffen worden, die sich hauptsächlich nach der Häufigkeit der Intervention in der ambulanten Allgemeinpraxis richtete. Zudem sollten diagnostische und therapeutische Empfehlungen gleich häufig vorkommen [20]. Unsere Studienteilnehmer hingegen wurden angehalten, die Relevanz für ihre tägliche Arbeit als Hauptkriterium zu 
verwenden, was die Unterschiede der Themenwahl teilweise erklärt.

Die fünf am häufigsten vorgeschlagenen Interventionen aus unserer Studie figurieren alle auf "Choosing wisely"-Listen verschiedener Fachgesellschaften, wie sie auf der Webseite der "Choosing wisely"-Kampagne publiziert sind [14]. Wir nehmen aber an, dass nicht die Kenntnis dieser Listen unsere Studienteilnehmer zu ihrer Themenauswahl motivierte, sondern dass - wie in der Aufgabenstellung formuliert - die Perspektive der Praxisarbeit dafür ausschlaggebend war. Daraus folgern wir, dass die Vorschläge von niedergelassenen Allgemeinärzten zu unangemessenen Interventionen (generiert aus ihrer Praxiserfahrung) nicht nur innerhalb unserer kleinen Studienpopulation relevant für ihre Arbeit sind, sondern auch für grössere Populationen gültig sein könnten (Einschränkungen: siehe weiter unten).

Der Fokus der Allgemeinpraktiker lag auf (Über-)Diagnose im Vergleich zu (Über-)Behandlung: Von unangemessenen Check-up-Untersuchungen, EKG's, Laboranalysen, Arthroskopien bis zu Magnetresonanztomogrammen (MRI's) des Neurokraniums, dies bei Gesunden oder bei Patienten mit einer selbstlimitierenden, nicht bedrohlichen Erkrankung.

Zu der am häufigsten genannten unangemessenen Abklärung - eine ungezielte Check-up Untersuchung - gibt es eine solide Datengrundlage, dass damit keine relevante Verbesserung klinischer Outcomes erreicht werden kann: Eine Cochrane-Review von 2012 fand keine Reduktion von Morbidität oder Mortalität durch Check-up Untersuchungen, weder über alle Ursachen noch für Krebs- oder Herzkreislauferkrankungen, hingegen stieg die Zahl neu gestellter Diagnosen (Überdiagnosen) an [21]. Eine dänische Arbeit von 2014 zeigte für Check-up Untersuchungen mit kardiovaskulärem Risikoscreening, sogar ergänzt durch wiederholte Beratung von identifizierten Risikoträgern, keine Effekte bezüglich Morbidität an koronarer Herzkrankheit, Hirnschlag oder Mortalität [22].

Hinsichtlich der am zweithäufigsten genannten unangemessenen Untersuchung rät die US-Amerikanische Preventive Service Task Force (USPSTF) aufgrund mangelnder Evidenzlage von der Durchführung von Ruhe- und Belastungs-EKG's bei 
asymptomatischen Individuen ab, und empfiehlt, dies den Patienten so zu kommunizieren [23]. Die Evidenzlage zur dritthäufigsten Nennung - Cholesterinanalysen und/oder Statintherapie im höheren Lebensalter - wird aktuell kontrovers diskutiert. Es bestehen zumindest Zweifel, ob das Verhältnis von Nutzen und Risiken im höheren Lebensalter [24].

Betrachtet man die "Choosing wisely"-Listen der verschiedener Fachgesellschaften, so sind Empfehlungen zur Diagnostik im Vergleich zu solchen zur Therapie etwa gleich häufig vertreten [14]. Die Absicht dahinter könnte sein, dadurch eine bessere Akzeptanz unter den Anwendern - inklusive Patienten - zu erreichen. Denn in der ursprünglichen Choosing wisely - Bewegung in den USA wurde schon früh festgestellt, dass Massnahmen gegen unangemessene Versorgung auf Widerstand stossen, weil sie nicht den traditionellen Konsumgewohnheiten entsprechen [25]. Erste Studien zur Wirkung zeigen, dass sich die Überdiagnostik in bestimmten Bereichen im Prä-Post-Vergleich nicht verändert haben: zum Beispiel inadäquate Knochendichtemessungen als Osteoporose-Screening [26], oder die Antibiotikaverschreibung bei akuten Sinusitiden [27]. Ähnliche fehlende Veränderungen wurden auch in der Schweiz vom Swiss Medical Board (eine Instanz, welche die Evidenz zu umstrittenen Interventionen untersucht und Empfehlungen abgibt) zum Thema Prostatakrebs-Screening durch PSA-Tests festgestellt [28]. Der Verzicht auf eine Intervention weckt offenbar bei Patienten Bedenken, dass Leistungen aus ökonomischen Gründen rationiert werden, oder wird als Entwertung wahrgenommen [29]. Eine sorgfältige Kommunikation zu Nutzen und Schaden (als Rationale bei der Diskussion, ob einer Intervention besser zu unterlassen sei) zwischen Patient und Arzt ist also entscheidend. Es kann nicht genug betont werden, wie wichtig die diesbezügliche Aus-, Weiter- und Fortbildung der Ärztinnen und Ärzte ist, damit sie diese Aufgabe kompetent wahrnehmen können [30]. Von grosser Bedeutung für die Dissemination der "Choosing wisely"-Philosophie, und deren erfolgreicher Umsetzung, wird sein, wie weit sich Allianzen zwischen Anbietern, Konsumenten, Ärzten und Politikern schmieden lassen [31].

Als Stärke unseres Ansatzes sehen wir das Einholen von Beiträgen, auf welche 
Interventionen besser zu verzichten sei, direkt von der Basis der niedergelassenen Hausärzte. Themen, die aus diesen Reihen stammen, könnten bezüglich Akzeptanz und Dissemination im Vergleich zu Experten-gesteuerten Listen Vorteile bringen: Ein höherer Identifikationsgrad mit einer Empfehlung kann sich in eine höhere Adhärenz ihr gegenüber übersetzen - ein Phänomen, das aus der Literatur zu Leitlinien bekannt ist [32]. Unsere Arbeit will nicht etwa die Relevanz der bestehenden "Smarter medicine"-Empfehlungen in Frage stellen, sondern neue Perspektiven zugunsten einer verbesserten Implementierung und Akzeptanz aufzeigen. Erfreulicherweise ist die SGAIM bereits dieser Einladung gefolgt, und plant mit uns zusammen die Erweiterung der "Smarter medicine" - Liste für die ambulante AIM für 2017, unter stärkerem Einbezug der niedergelassenen Ärzte.

Eine Limitation unserer Studie sehen wir im methodischen Ansatz: Die Empfehlungen aus unserer Studie sind aufgrund der Studienpopulation (Grösse, Zusammensetzung) nicht auf die gesamte (Schweizerische) Hausärzteschaft generalisierbar. Das Ziel der Studie war dementsprechend auch nicht die Bereitstellung allgemeingültiger Empfehlungen, sondern die Darstellung eines innovativen Ansatzes als Ausgangspunkt für eine Weiterentwicklung von Empfehlungen.

Eine weitere Limitation betrifft die extrem niedrige Rücklaufquote aus der JHaS-Befragung, deren Resultate sicher nicht repräsentativ für die Jungen Hausärzte sind. Eine mögliche Ursache für diese niedrige Rücklaufquote könnte sein, dass in der Phase zur Weiterbildung zum Facharzt und der Praxisgründung andere Interessen im Vordergrund sind, als auf welche Interventionen besser zu verzichten sei.

\section{Konklusion}

Es ist gleichermassen wichtig, potentiell unangemessene medizinische Interventionen aus der Sicht der Anwender (praktizierende Ärzte) zu explorieren, wie auch Daten dazu in der Perspektive des Gesundheits-Versorgungssystems zu sammeln. Die in unserer Studie vorgeschlagenen Interventionen könnten, optimalerweise in Zusammenarbeit mit 
der offiziellen "Smarter medicine" Kampagne, zu weiteren Empfehlungen führen, was in der Grundversorgung besser zu unterlassen sei.

Entscheidend für den Erfolg ist die "letzte Meile" der adäquaten Kommunikation: Einerseits die Kommunikation zwischen Ärzten und ihren Patienten hinsichtlich Verzicht auf eine unangemessene Intervention, und andrerseits diejenige zwischen Versorgungsforschern und praktizierenden Ärzten, auf dass letztere ihr neues Wissen zu unangemessenen Interventionen in die Tat umsetzen.

\section{Tabellen}

\section{Empfehlung}

Durchführen einer bildgebenden Diagnostik in den ersten sechs Wochen bei Patienten mit unspezifischen Lumbalgien

Messung des Prostata-spezifischen Antigens (PSA) zwecks Prostatakrebs-Screening ohne eine Diskussion von Risiko und Nutzen

Verschreiben von Antibiotika gegen unkomplizierte Infekte der oberen Luftwege

Durchführen eines präoperativen Thorax-Röntgenbildes, ausser bei Verdacht auf eine intrathorakale Pathologie

Weiterführen einer Langzeit-Pharmakotherapie bei gastrointestinalen Symptomen mit

Protonen-Pumpenblockern ohne Reduktion auf die tiefste wirksame Dosis

\section{Tabelle 1}

Die Empfehlungen der Schweizerischen Gesellschaft für Innere Medizin (SGAIM) im Rahmen der "Smarter Medicine"-Kampagne (2015), welche fünf Interventionen in der ambulanten Allgemeinen Inneren Medizin zu vermeiden sind [19][20] 


\begin{tabular}{|l|c|}
\hline Empfehlung & $\mathrm{n}^{*}$ \\
\hline Check-up-Untersuchung, vor allem bei jüngeren / asymptomatischen Individuen $^{\text {a }}$ & 5 \\
\hline Ruhe- oder Belastungs-EKG bei asymptomatischen Individuen $^{\text {b }}$ & 5 \\
\hline $\begin{array}{l}\text { Cholesterin-Analyse bei Individuen älter als 75 oder Statintherapie in der Primärprävention } \\
\text { und/oder hohem Alter }\end{array}$ & 5 \\
\hline Arthroskopie oder MRI des Kniegelenks nach Distorsion, ohne Instabilität oder Blockade $^{\text {d }}$ & 4 \\
\hline Bildgebende Diagnostik bei Kopfweh ohne Alarmzeichen $^{\text {e }}$ & 3 \\
\hline
\end{tabular}

\section{Tabelle 2}

Die fünf häufigsten Empfehlungen der niedergelassenen Ärzte in unserer Studie, welche Interventionen in der ambulanten Allgemeinen Inneren Medizin zu vermeiden sind, geordnet nach Häufigkeit der Nennung.

* Anzahl Gruppen, welche diese Empfehlung parallel entwickelt haben

In der Listen-Sammlung der Choosing-wisely-Webseite [14] finden sich diese Empfehlungen in den Listen von folgenden Gesellschaften (mit Jahr der Publikation):

a. American Society of General Internal Medicine (2013); The College of Family Physicians of Canada (2014)

b. American Society of Family Physicians (2012); American Society of Cardiology (2012); Canadian

Cardiovascular Society (2014), National Institute for Health and Care Excellence NICE (2015)

c. Society of Post-acute and Long-term Care Medicine (2013)

d. American Medical Society for Sports Medicine (2014)

e. American college of Radiology (2012); American Headache Society (2013); Canadian Association of Radiologists (2014); National Institute for Health and Care Excellence NICE (2015) 
Empfehlung

Routine-Urinanalysen ohne das Vorliegen von Symptomen

Steroidinfiltrationen bei unspezifischen Lumbalgien

Antibiotikatherapie bei akuter Sinusitis

Obere Endoskopie bei Dyspepsie ohne Alarmzeichen

Computertomographie bei akuter Appendicitis

Routine-Bildgebung bei Distorsionstrauma des oberen Sprungelenkes

Messung von Eisenparametern bei normalem Hämoglobin und in Abwesenheit von Symptomen

Routine-Kombination von Protonenpumpenblockern mit nicht-steroidalen Antirheumatica

C-reaktives Protein und Blutbild als Routinediagnostik bei akuten Infektionen

Langzeitbehandlung mit Psychopharmaka ohne gleichzeitige Gesprächs-Psychotherapie

Antihypertensiva ohne Adaptation ans Lebensalter

\section{Tabelle 3}

Die weniger häufig (von jeweils zwei Gruppen entwickelten) Empfehlungen der niedergelassenen Ärzte in unserer Studie, welche Interventionen in der ambulanten Allgemeinen Inneren Medizin zu vermeiden sind

\section{Referenzen}

[1] A.D. Kaiser, Effect of Tonsillectomy on General Health in five thousand Children, J Am Med Assoc 78 (1922) 1869-1873.

[2] L. Arkin, A Study of Conditions Found in One Thousand Cases of Tonsillectomy and Adenotomy, The Boston Medical and Surgical Journal 192 (1925) 597-602.

[3] J.A. Glover, The Incidence of Tonsillectomy in School children. Int J Epidemiol 37 (1938) 9-19.

[4] N. Miller, Hysterectomy - Therapeutic Necessity or Surgical Racket? J Obstet Gynecol 51 (1946) 804-810.

[5] R.P. Bolande, Ritualistic Surgery - Circumcision and Tonsillectomy, New England Journal of Medicine 280 (1969) 591-596.

[6] J. Wennberg, A. Gittelsohn, Small Area Variations in Health Care Delivery: A Population-Based Health Information System Can Guide Planning and Regulatory Decision-Making, Science 182 (1973) 1102-1108. 
[7] M.R. Chassin, R.W. Galvin, and the National Roundtable on Health Care Quality, IOM: The Urgent Need to Improve Health Care Quality: Institute of Medicine National Roundtable on Health Care Quality, JAMA 280 (1998) 1000-1005.

[8] Institute of Medicine: Crossing the Quality Chasm: A New Health System for the 21st Century, Washington, D.C., National Academy Press (2001)

[9] R. Moynihan, R. Smith, Too Much Medicine? Almost Certainly, BMJ 324 (2002) 859

[10] S. Brownlee, Overtreated: Why Too Much Medicine Is Making Us Sicker and Poorer, Bloomsbury USA, $1^{\text {st }}$ edition (2008)

[11] I. Heath, It is not Wrong to Say No, BMJ 338 (2009) b2529

[12] G. Mulley, Stop the Medicalization of Old Age, BMJ 344 (2012) e803

[13] P. Glasziou, R. Moynihan, T. Richards, F. Godlee, Too Much Medicine; too Little Care. Time to Wind Back the Harms of Overdiagnosis and Overtreatment, BMJ 346 (2013) f1271.

[14] http://www.choosingwisely.org (letzter Zugriff 30.8.2016)

[15] Medical Professionalism Project: Medical Professionalism in the New Millennium: A Physician Charter, Ann Intern Med 136 (2002) 243-246.

[16] E.G. Campbell, S. Regan, R.L. Gruen, T.G. Ferris, S.R. Rao, P.D. Cleary, D. Blumenthal, Professionalism in Medicine: Results of a National Survey of Physicians, Ann Intern Med 147 (2007) 795-802.

[17] The ABIM Foundation: Unnecessary Tests and Procedures In the Health Care System. What Physicians Say About The Problem, the Causes, and the Solutions Results from a National Survey of Physicians (2014)

[18] D. Wolfson, J. Santa, L. Slass, Engaging Physicians and Consumers in Conversations About Treatment Overuse and Waste: A Short History of the Choosing Wisely Campaign. Acad Med 89 (2014) 990-995.

[19] http://www.smartermedicine.ch (letzter Zugriff 30.8.2016)

[20] K. Selby, J.M. Gaspoz, N. Rodondi, S. Neuner-Jehle, A. Perrier, A. Zeller, J. Cornuz, Creating a List of Low-Value Health Care Activities in Swiss Primary Care, JAMA Intern Med 175 (2015) 640-2. 
[21] L.T. Krogsbøll, K.J. Jørgensen, C. Grønhøj, P.C. Gøtzsche, General Health Checks in Adults for Reducing Morbidity and Mortality from Disease, Cochrane Database for Systematic Reviews (2012) Issue 10, Art. No.: CD009009

[22] T. Jørgensen, R.K. Jacobsen, U. Toft, M. Aadahl, C. Glümer, C Pisinger et al, Effect of Screening and Lifestyle Counselling on Incidence of Ischaemic Heart Disease in General Population: Inter99 Randomised Trial, BMJ 348 (2014) g3617

[23] http://www.uspreventiveservicestaskforce.org (letzter Zugriff 30.8.2016)

[24] M.C. Odden, M.J. Plechter, P.G. Coxson, D. Thekkethala, D. Guzman, D. Heller, L. Goldman, K. Bibbins-Domingo, Cost-Effectiveness and Population Impact of Statins for Primary Prevention in Adults Aged 75 Years or Older in the United States, Annals Intern Med 163 (2015) 533-541

[25] J. Santa, Communicating Information About "What Not to Do" to Consumers, BMC Med Informatics Decision Making 13 (Suppl 3)(2013) S2

[26] E.C. Lasser, E.R. Pfoh1, H.Y. Chang, K.S. Chan, J.C. Bailey, H. Kharrazi, J.P. Weiner, S.M. Dy, Has Choosing Wisely ${ }^{\circledR}$ Affected Rates of Dual-Energy X-ray Absorptiometry Use? Osteoporos Int (2016) DOI 10.1007/s00198-016-3511-0

[27] A.L. Sharp, M.H. Klau, D. Keschner, E. Macy, T. Tang et al. Low Value Care for Acute Sinusitis Encounters: Who's Choosing Wisely? Am J Manag Care 21 (2015) 479-85

[28] K. Eichler, S. Hess, M. Riguzzi, Ü. Can, U. Brügger, Impact Evaluation of Swiss Medical Board Reports on Routine Care in Switzerland: a Case Study of PSA Screening and Treatment for Rupture of Anterior Cruciate Ligament, Swiss Med Wkly 145 (2015) w14140

[29] J. Schuling, H. Gebben, L.J. Veehof, F.M. Haaijer-Ruskamp, Deprescribing Medication in Very Elderly Patients with Multimorbidity: The View of Dutch GPs. A Qualitative Study, BMC Fam Pract 13 (2012) 56.

[30] S. Neuner-Jehle, O. Senn, O. Wegwarth, T. Rosemann, J. Steurer, How do Family Physicians Communicate About Cardiovascular Risk? Frequencies and Determinants of Different Communication Formats, BMC Family Practice 12 (2011) 15

[31] J.M. Gaspoz, Smarter Medicine: Do Physicians Need Political Pressure to Eliminate Useless Interventions? Swiss Med Wkly 145 (2015) w14125

[32] M.D. Cabana, C.S. Rand, N.R. Powe, A.W. Wu, M.H. Wilson, P.A.C. Abboud, H.R. Rubin, Why Don't Physicians Follow Clinical Practice Guidelines? A Framework for Improvement, JAMA 282 (1999) 1458-1465 